\title{
EVOLUÇÃO DOS QUESTIONÁRIOS OSWESTRY 2.0 E DO COMPONENTE FÍSICO (PCS) DO SF-36 DURANTE O PRIMEIRO ANO DE PÓS-OPERATÓRIO DE ARTRODESE DA COLUNA LOMBAR EM DOENÇAS DEGENERATIVAS
}

\author{
EVOLUTION OF OSWESTRY 2.0 QUESTIONNAIRE AND THE PHYSICAL COMPONENT (PCS) \\ OF SF-36 DURING THE FIRST POSTOPERATIVE YEAR OF LUMBAR SPINE \\ FUSION IN DEGENERATIVE DISEASES
}

\author{
EVOLUCIÓN DEL CUESTIONARIO OSWESTRY 2.0 Y DEL COMPONENTE FÍSICO (PCS) \\ DEL SF-36 DURANTE EL PRIMER AÑO DE POSTOPERATORIO DE FUSIÓN \\ LUMBAR EN ENFERMIDADES DEGENERATIVAS
}

Alexandre Henrique Silveira Bechara ${ }^{1}$, Guilherme Rebechi Zuiani ${ }^{1}$, Marcelo Ítalo Risso Neto ${ }^{1}$, Paulo Tadeu Maia Cavali', Ivan Guidolin Veiga', Wagner PasqualinI ${ }^{1}$, Elcio Landim ${ }^{1}$

\begin{abstract}
RESUMO
Objetivo: Avaliar a melhora clínica dos pacientes submetidos a descompressão e artrodese lombar póstero-lateral por doença degenerativa, avaliando a evolução dos escores do questionário Oswestry 2.0 e pelo componente físico (PCS) da escala SF-36. Métodos: Estudo prospectivo com 19 pacientes consecutivos com diagnóstico de doença degenerativa de disco (hérnia de disco, estenose lombar ou espondilolistese degenerativa) submetidos à descompressão e artrodese póstero-lateral. Todos os pacientes incluídos em nosso estudo responderam aos questionários Oswestry 2.0 e SF-36 em cinco momentos diferentes: no período préoperatório e após 45, 90, 180 e 360 dias de cirurgia. Resultados: Os escores dos questionários Oswestry 2.0 e do componente físico (PCS) do SF-36 dos 19 pacientes mostraram melhora significativa ( $p<0,001$ e $p=0,004$ respectivamente) ao longo de um ano de seguimento pós-operatório. Não houve diferença significativa de melhora desses escores quando feita comparação entre os diagnósticos (hérnia de disco, estenose lombar ou espondilolistese degenerativa), o sexo dos pacientes e entre os indivíduos com idade até cinquenta anos e aqueles acima de cinquenta anos. Conclusão: Houve uma melhora dos escores nos questionários de Oswestry 2.0 e do componente físico (PCS) do SF-36 nos pacientes com doença degenerativa da coluna lombar sem resposta ao tratamento conservador, que foram submetidos a tratamento cirúrgico com descompressão e artrodese após um ano de seguimento pós-operatório.
\end{abstract}

Descritores: Deslocamento do disco intervertebral; Estenose espinal; Espondilolistese, Fusão vertebral.

\begin{abstract}
Objective: Assess the clinical improvement of patients undergoing decompression and posterolateral lumbar arthrodesis for degenerative diseases, evaluating the evolution of the scores of Oswestry 2.0 questionnaire and the physical component (PCS) of the SF-36 scale. Methods: Prospective study of 19 patients with degenerative disc disease (disc herniation, lumbar stenosis or degenerative spondylolisthesis) that underwent decompression and posterolateral arthrodesis. All patients included in our study answered the questionnaire Oswestry 2.0 and the PCS of SF-36 at five different occasions: in the preoperative period and 45, 90, 180 and 360 days after surgery. Results: The scores of Oswestry 2.0 questionnaire and the physical component (PCS) of the SF-36 of the patients showed significant improvement $(p<0.001$ and $p=0.004$ respectively) over a year of postoperative follow-up. There was no significant difference in improvement in these scores when comparing the diagnoses made (disc herniation, lumbar stenosis or degenerative spondylolisthesis), sex of patients, and individuals aged up to fifty years and those over fifty years. Conclusion: There was an improvement in Oswestry scores and physical component (PCS) of the SF-36 in patients with degenerative lumbar spine unresponsive to conservative treatment that underwent surgical decompression and arthrodesis after one year of postoperative follow-up.
\end{abstract}

Keywords: Intervertebral disc displacement; Spinal stenosis; Spondylolisthesis; Spinal fusion.

\section{RESUMEN}

Objetivo: Evaluar la mejoría clínica de los pacientes sometidos a descompresión y artrodesis lumbar posterolateral para las enfermedades degenerativas, valoración de la evolución de las puntuaciones del cuestionario Oswestry 2.0 y el componente físico (PCS) de la escala SF-36. Métodos: Evaluación de 19 pacientes con enfermedad degenerativa del disco (hernia de disco, estenosis lumbar o espondilolistesis degenerativa) sometidos a descompresión y artrodesis posterolateral. Todos los pacientes incluidos en nuestro estudio respondieron al cuestionario de Oswestry 2.0 e al SF-36 en cinco ocasiones distintas: Período preoperatorio y 45, 90, 180 y 360 días después de la cirugía. Resultados: Las puntuaciones del cuestionario Oswestry y el componente físico (PCS) de la SF-36 de los 19 pacientes mostraron una mejoría significativa

1. Universidade Estadual de Campinas (Unicamp), Campinas, SP, Brasil.

Trabalho realizado na Disciplina de Cirurgia da Coluna do Departamento de Ortopedia eTraumatologia da Universidade Estadual de Campinas (Unicamp), Campinas, SP, Brasil. Correspondência: Rua Tessália Vieira de Camargo, 126. Cidade Universitária Zeferino Vaz. Campinas, SP. Brasil.13083. mrisso@mpc.com.br 
( $p<0,001$ y $p=0,004$, respectivamente) a lo largo de un año de seguimiento postoperatorio. No hubo diferencias significativas en la mejoría de los puntajes cuando se compararon los diagnósticos realizados (hernia de disco, estenosis lumbar y espondilolistesis degenerativa), sexo de los pacientes e individuos hasta la edad de cincuenta años y los mayores de cincuenta años. Conclusión: Se observó una mejoría en las puntuaciones en los cuestionarios de Oswestry 2.0 y el componente físico (PCS) de la SF-36 en pacientes con enfermedad degenerativa de la columna lumbar que no respondieron al tratamiento conservador y fueron sometidos a cirugía de descompresión y artrodesis en el seguimiento después de un año de la cirugía.

Descriptores: Desplazamiento del disco intervertebral; Estenosis espinal; Espondilolistesis; Fusión vertebral.

\section{INTRODUÇÃO}

A dor lombar é um dos problemas de saúde mais comuns da atualidade, causando considerável incapacidade, faltas ao trabalho e gastos ao Sistema de Saúde. Webb et al. ${ }^{1}$ relatou que a dor lombar afeta aproximadamente um quarto dos adultos em um intervalo de um mês; sendo essa definida como crônica temporalmente pela duração superior a três semanas. Andersson ${ }^{2}$ em estudo epidemiológico relatou que $50-80 \%$ das pessoas irão apresentar pelo menos um episódio de dor lombar durante a vida. Cassidy et al. ${ }^{3}$ relataram que num período aleatório de seis semanas, $72 \%$ dos adultos de uma população em geral irão relatar um episodio de dor lombar, e desses, $11 \%$ irão apresentar alguma incapacidade funcional.

É importante lembrar que a dor lombar não é um diagnóstico, por isso, não pode ser considerada como a causa de alteração anatômica e sim um sintoma. A causa exata da dor na maioria desses episódios não consegue ser definida, sendo então classificadas como "dor lombar inespecífica". Dentre as causas específicas da lombalgia incluem-se as apresentações da doença degenerativa discal (DDD) como hérnia de disco, estenose de canal, espondilolistese e espondiloartrose. As causas especificas correspondem a aproximadamente $20 \%$ dos casos. ${ }^{4}$

O processo natural de evolução da doença degenerativa discal (DDD) foi descrito por Yong-Hing e Kirkaldy-Willis ${ }^{5}$ em três estágios: disfunção, instabilidade e estabilização. A hérnia discal costuma ocorrer nos estágios de disfunção e instabilidade, enquanto a estenose de canal no estágio de instabilidade avançada e inicio do processo de estabilização.

Além da dificuldade do diagnóstico e da indicação do melhor tipo de tratamento na doença degenerativa, existe também a dificuldade em avaliar os resultados do tratamento realizado. Diversas escalas de avaliação como Oswestry, SF-36, Roland Morris, critérios de Brodsky e escalas visuais analógicas de dor foram desenvolvidas para a avaliação subjetiva dos resultados pós-operatórios. ${ }^{6,7} \mathrm{O}$ objetivo desse estudo é avaliar se houve meIhora funcional nos pacientes com doença degenerativa lombar submetidos a descompressão e artrodese instrumentada por via posterior, por meio da avaliação dos questionários Oswstry $2.0^{8}$ e pelo componente físico (PCS) da escala SF-36..$^{9,10}$

\section{PACIENTES E MÉTODOS}

Este é um estudo prospectivo feito pela avaliação subjetiva, por meio de dois questionários, Oswestry 2.0 e componente físico (PCS) do SF-36, dos pacientes submetidos a tratamento cirúrgico por doença degenerativa lombar.

Foram avaliados dezenove pacientes consecutivos do Ambulatório de Coluna do Hospital de Clínicas da Universidade Estadual de Campinas (Unicamp) no período de maio de 2010 até setembro de 2011 submetidos a tratamento cirúrgico com descompressão e artrodese posterolateral de coluna lombar após falha do tratamento conservador pelo período mínimo de 12 semanas. Em todos os pacientes foi utilizado enxerto ósseo autólogo local (lâmina e processo espinhoso).

Todos os pacientes foram seguidos pelo período de um ano. Os questionários Oswestry 2.0 e SF-36 foram respondidos em cinco momentos diferentes: no período pré-operatório e após 45, 90, 180 e 360 dias de cirurgia. As questões eram lidas e explicadas aos pacientes em todas as avaliações pelo mesmo examinador. Esse examinador não participou do procedimento cirúrgico.

Foram aplicados testes Kolmogorov-Smirnov ${ }^{11}$ para avaliar a normalidade de distribuição dos dados de idade e das escalas de Owestry 2.0 e componente físico (PCS) do SF-36 a cada momento de avaliação e aplicadas análises de variâncias (ANOVA) com medidas repetidas para comparação das escalas entre os momentos de avaliação, seguidas de comparações múltiplas de Bonferroni ${ }^{12}$ para comparar os momentos dois a dois. ${ }^{11,12}$

Foram calculadas as medidas de variação do Oswestry 2.0 [inicial - final (360 dias)] e variação do componente físico de qualidade de vida [final (360 dias) - inicial] e descritas as medidas de variação segundo diagnósticos, sexos e faixas etárias com uso de medidas resumo (média, desvio padrão, mediana, mínimo e máximo). As variações nas escalas foram comparadas entre os diagnósticos com uso de análises de variâncias (ANOVA) e entre sexos e faixas etárias com uso de testes $t$ Student. ${ }^{11}$ Foram apresentados os perfis médios dos escores de Owestry 2.0 e dos escores do componente físico (PCS) do SF-36 com os respectivos erros padrões e os testes foram realizados com nível de significância de $5 \%$.

\section{RESULTADOS}

Foram avaliados dezenove pacientes sendo treze $(68,4 \%)$ do sexo feminino e seis $(31,6 \%)$ do sexo masculino. A média de idade dos pacientes foi de 49 anos (DP $=12$ anos). Os diagnósticos dos pacientes submetidos a tratamento cirúrgico foram hérnia discal $(52,6 \%)$, estenose de canal lombar $(31,6 \%)$ e espondilolistese degenerativa (15,8\%). (Tabela 1)

No período pré-operatório, a média do resultado do questionário de Oswestry foi de 49,89\% (DP de 15,52\%) e do componente físico (PCS) do SF-36 foi de 35,11 pontos (DP de 7,35 pontos). Após 45 dias da realização da cirurgia, a média do resultado do questionário de Oswestry foi de 20,42\% (DP de 16,04\%) e do PCS foi de 41,79 pontos (DP de 7,50 pontos). Após 90 dias de pós-operatório, a média da pontuação do questionário de Oswestry foi 21,74\% (DP de $13,86 \%$ ) e do PCS foi de 40,47 pontos (DP de 8,83 pontos). Avaliando o questionário de Oswestry em 180 dias de pós-operatório, a média da pontuação foi de 19,63\% (DP de 17,14\%) e do PCS foi de 43,47 pontos (DP de 9,91 pontos). Por último, com 360 dias de pós-operatório, a média de pontuação do questionário de Oswestry foi de $17,42 \%$ (DP de 18,18\%) e do PCS a média foi de 44,58 pontos (DP de 9,47 pontos). (Tabela 2) Houve diferença com significância estatística $(p<0,001)$ do escore do questionário Oswestry entre o

Tabela 1. Descrição das características gerais dos pacientes.

\begin{tabular}{c|c|c}
\hline Variável & N & $\%$ \\
\hline Diagnóstico & & \\
\hline Hérnia & 10 & 52,6 \\
\hline Estenose & 6 & 31,6 \\
\hline Espondilolistese degenerativa & 3 & 15,8 \\
\hline Sexo & & \\
\hline Feminino & 13 & 68,4 \\
\hline Masculino & 6 & 31,6 \\
\hline Idade & & \\
\hline média(DP) & $49,1(11,9)$ & \\
\hline Total & 19 & 100 \\
\hline
\end{tabular}


período pré-operatório e os demais períodos da avaliação ao longo de um ano de seguimento. (Figura 1) O escore médio do componente físico (PCS) do SF-36 aumenta com significância estatística $(p=0,004)$ entre o período pré-operatório e os demais momentos da avaliação no intervalo de um ano. (Figura 2)

Avaliando a pontuação do questionário de Oswestry, a comparação do período pré-operatório com os períodos 45, 90, 180 e 360 dias de pós-operatório mostrou uma melhora de 29,47\% em média no questionário de 45 dias (desvio-padrão de 5,12\%); de 28,16\% (desvio-padrão de 5,16\%) no questionário de 90 dias; de 30,26\% (desvio-padrão de 5,14\%) no questionário de 180 dias e de $32,47 \%$ (desvio-padrão de 5, 20\%) no questionário de 360 dias. A porcentagem de melhora apresentou significância estatística $(p<0,001)$. A comparação dos escores obtidos com os questionários de Oswestry e com o componente físico do SF-36 entre os diferentes períodos pós-operatórios em que foram aplicados não apresentou diferença com significância estatística. (Tabela 3)

A análise do componente físico (PCS) do SF-36 mostrou significância estatística na comparação dos valores dos questionários do período pré-operatório em relação ao questionário aplicado após 360 dias da cirurgia $(p=0,025)$. A diferença nesse período foi de 9,47 pontos (desvio-padrão de 2,71 pontos). (Tabela 3)

A média da melhora do componente físico (PCS) do SF-36 é de 9,47 pontos (DP de 11,79 pontos).

A comparação dos resultados obtidos nos mesmos períodos por meio dos questionários de Oswestry 2.0 e do componente físico do SF-36 entre os sexos (masculino e feminino), os diferentes diagnósticos (hérnia de disco, estenose de canal e espondilolistese degenerativa) e faixa etária (até 50 anos e acima de 50 anos) não mostrou diferença com significância estatística nos valores obtidos. (Tabelas 4 e 5)

Tabela 2. Descrição do Oswetry 2.0 e componente físico (PCS) do SF-36 segundo os momentos de avaliação e resultado dos testes comparativos.

\begin{tabular}{|c|c|c|c|c|c|c|c|c|}
\hline Variável & Momento & Média & DP & Mediana & Mínimo & Máximo & $\mathbf{N}$ & p \\
\hline \multirow{5}{*}{$\begin{array}{c}\text { Oswestry } \\
(\%)\end{array}$} & pré & 49,89 & 15,52 & 46 & 26 & 78 & 19 & \multirow{5}{*}{$<0,001$} \\
\hline & 45 dias & 20,42 & 16,04 & 16 & 0 & 52 & 19 & \\
\hline & 90 dias & 21,74 & 13,86 & 24 & 0 & 56 & 19 & \\
\hline & 180 dias & 19,63 & 17,14 & 16 & 0 & 64 & 19 & \\
\hline & 360 dias & 17,42 & 18,18 & 10 & 0 & 52 & 19 & \\
\hline \multirow{5}{*}{$\begin{array}{c}\text { Componente } \\
\text { físico (PCS) }\end{array}$} & pré & 35,11 & 7,35 & 34 & 24 & 47 & 19 & \multirow{5}{*}{0,004} \\
\hline & 45 dias & 41,79 & 7,50 & 41 & 30 & 55 & 19 & \\
\hline & 90 dias & 40,47 & 8,83 & 40 & 22 & 54 & 19 & \\
\hline & 180 dias & 43,47 & 9,91 & 44 & 24 & 62 & 19 & \\
\hline & 360 dias & 44,58 & 9,47 & 46 & 29 & 57 & 19 & \\
\hline
\end{tabular}

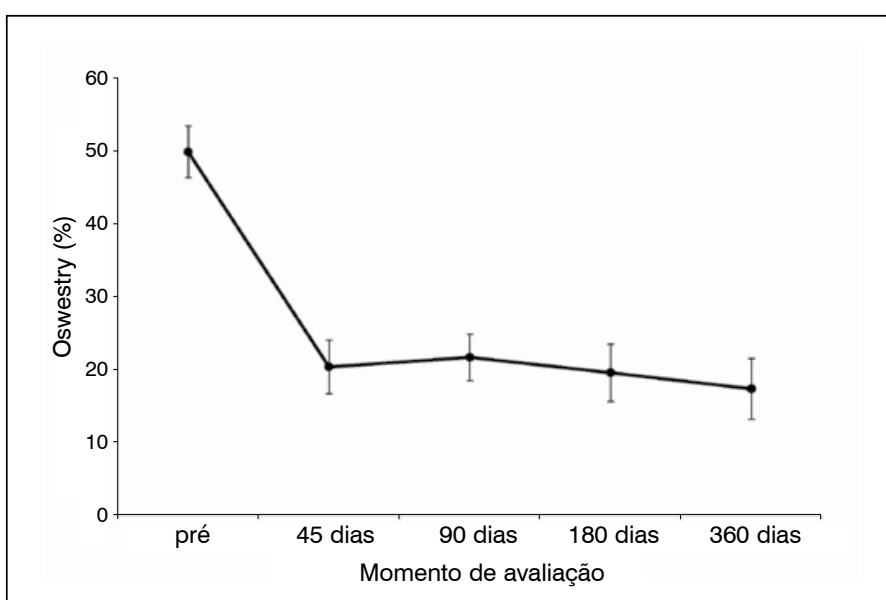

Figura 1. Perfil médio do Oswestry 2.0 ao longo de um ano de pós-operatório.

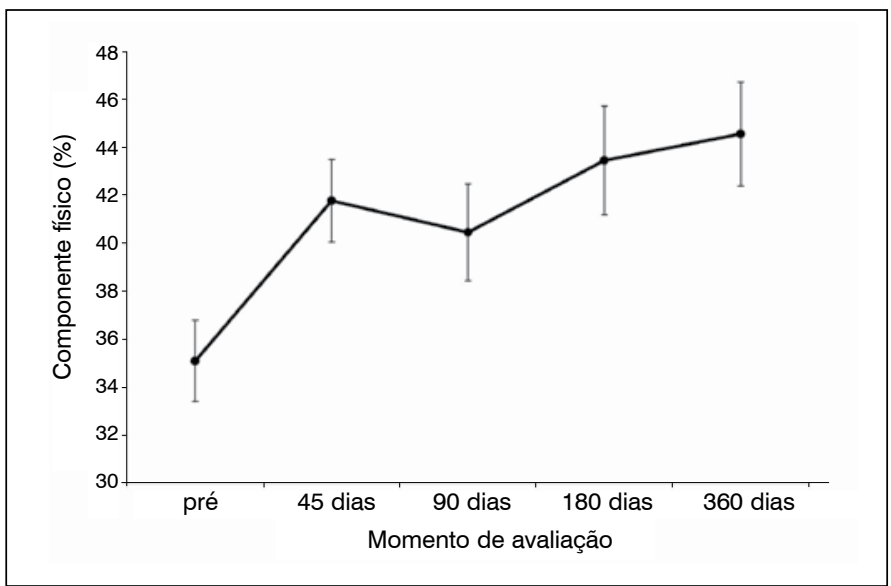

Figura 2. Perfil médio do escore do componente físico (PCS) d0 SF-36 ao longo de um ano de pós-operatório.

Tabela 3. Resultado das comparações múltiplas das escalas entre os momentos de avaliação.

\begin{tabular}{|c|c|c|c|c|c|}
\hline Variável & Comp & ação & Diferença média & Erro Padrão & $\mathbf{p}$ \\
\hline \multirow{10}{*}{$\begin{array}{c}\text { Oswestry } \\
(\%)\end{array}$} & Pré - & 45 & 29,47 & 5,12 & $<0,001$ \\
\hline & Pré - & 90 & 28,16 & 5,16 & $<0,001$ \\
\hline & Pré - & 180 & 30,26 & 5,14 & $<0,001$ \\
\hline & Pré - & 360 & 32,47 & 5,20 & $<0,001$ \\
\hline & $45-$ & 90 & $-1,32$ & 3,21 & $>0,999$ \\
\hline & $45-$ & 180 & 0,79 & 4,73 & $>0,999$ \\
\hline & $45-$ & 360 & 3,00 & 4,32 & $>0,999$ \\
\hline & 90 - & 180 & 2,11 & 2,89 & $>0,999$ \\
\hline & 90 - & 360 & 4,32 & 3,71 & $>0,999$ \\
\hline & $180-$ & 360 & 2,21 & 3,42 & $>0,999$ \\
\hline \multirow{10}{*}{$\begin{array}{l}\text { Componente } \\
\text { físico (PCS) }\end{array}$} & Pré - & 45 & $-6,68$ & 2,68 & 0,226 \\
\hline & Pré - & 90 & $-5,37$ & 2,87 & 0,777 \\
\hline & Pré - & 180 & $-8,37$ & 2,76 & 0,072 \\
\hline & Pré - & 360 & $-9,47$ & 2,71 & 0,025 \\
\hline & $45-$ & 90 & 1,32 & 2,54 & $>0,999$ \\
\hline & $45-$ & 180 & $-1,68$ & 3,05 & $>0,999$ \\
\hline & $45-$ & 360 & $-2,79$ & 2,56 & $>0,999$ \\
\hline & 90 - & 180 & $-3,00$ & 1,79 & $>0,999$ \\
\hline & 90 - & 360 & $-4,11$ & 2,04 & 0,589 \\
\hline & $180-$ & 360 & $-1,11$ & 1,87 & $>0,999$ \\
\hline
\end{tabular}

\section{DISCUSSÃO}

A técnica de descompressão e artrodese de um segmento da coluna é um dos métodos de tratamento utilizado nos pacientes com diagnóstico de estenose ou hérnia de disco associada à instabilidade quando ocorre falha com o tratamento conservador. ${ }^{13}$ Essa modalidade de tratamento apresenta como vantagens a eliminação do movimento do segmento afetado, que pode ser uma das causas da dor, e a possibilidade de uma descompressão ampla das estruturas acometidas. ${ }^{14,15} \mathrm{O}$ enxerto ósseo para fusão da coluna pode ser obtido de fontes locais como facetas, lâminas e processo espinhoso, do ilíaco ou ainda de banco de osso (aloenxerto). ${ }^{16}$ 
Tabela 4. Descrição das variações dos escores Oswestry 2.0 (inicial - final) segundo diagnósticos, sexos e faixas etárias e resultado dos testes comparativos.

\begin{tabular}{c|c|c|c|c|c|c|c}
\hline Variável & Média & DP & Mediana & Mínimo & Máximo & $\mathbf{N}$ & $\mathbf{p}$ \\
\hline Diagnóstico & & & & & & & 0,700 \\
\hline Hérnia & 36,60 & 23,51 & 37 & -8 & 76 & 10 & \\
\hline Estenose & 26,33 & 24,93 & 18 & 8 & 76 & 6 & \\
\hline $\begin{array}{c}\text { Espondilolistese } \\
\text { degenerativa }\end{array}$ & 31,00 & 19,47 & 38 & 9 & 46 & 3 & \\
\hline $\begin{array}{c}\text { Sexo } \\
\text { Feminino }\end{array}$ & 28,23 & 21,22 & 24 & -8 & 76 & 13 & \\
\hline Masculino & 41,67 & 24,90 & 34 & 14 & 76 & 6 & \\
\hline Faixa etária & & & & & & & 0,240 \\
\hline Até 50 anos & 32,08 & 24,26 & 31 & -8 & 76 & 12 & \\
\hline 50 anos ou mais & 33,14 & 21,47 & 30 & 8 & 76 & 7 & \\
\hline Total & 32,47 & 22,66 & 30 & -8 & 76 & 19 & \\
\hline
\end{tabular}

Tabela 5. Descrição das variações dos escores do componente físico (PCS) do SF-36 (final - inicial) segundo diagnósticos, sexos e faixas etárias e resultado dos testes comparativos.

\begin{tabular}{c|c|c|c|c|c|c|c}
\hline Variável & Média & DP & Mediana & Mínimo & Máximo & $\mathbf{N}$ & $\mathbf{p}$ \\
\hline Diagnóstico & & & & & & & 0,318 \\
\hline Hérnia & 13,20 & 11,14 & 15 & -3 & 27 & 10 & \\
\hline Estenose & 6,83 & 13,92 & 9 & -15 & 22 & 6 & \\
\hline $\begin{array}{c}\text { Espondilolistese } \\
\text { degenerativa }\end{array}$ & 2,33 & 6,43 & 5 & -5 & 7 & 3 & \\
\hline $\begin{array}{c}\text { Sexo } \\
\text { Feminino }\end{array}$ & 8,92 & 10,94 & 7 & -5 & 27 & 13 & \\
\hline Masculino & 10,67 & 14,51 & 15 & -15 & 26 & 6 & \\
\hline Faixa etária & & & & & & & 0,679 \\
\hline Até 50 anos & 8,58 & 13,79 & 6 & -15 & 27 & 12 & \\
\hline 50 anos ou mais & 11,00 & 8,00 & 11 & -3 & 21 & 7 & \\
\hline \begin{tabular}{c} 
Total \\
\hline
\end{tabular} & 9,47 & 11,79 & 9 & -15 & 27 & 19 & \\
\hline
\end{tabular}

A instrumentação da coluna lombar no procedimento de artrodese é utilizada para aumentar a taxa de fusão, diminuir o tempo de imobilização e, com isso, melhorar os resultados cirúrgicos a longo prazo. ${ }^{17}$ No presente estudo, todos pacientes foram submetidos a instrumentação do(s) nível(is) degenerados com parafusos pediculares e foi utilizado enxerto autólogo local.

Existem várias maneiras de avaliar o sucesso do procedimento cirúrgico (retorno ao trabalho, presença de fusão sólida, redução da dor, status funcional e aplicação de questionários). O uso do grau de satisfação dos pacientes com o tratamento também tem sido utilizado como parâmetro para avaliação do resultado. ${ }^{18}$ Segundo Turner et al. ${ }^{19}$ a satisfação do paciente com o procedimento cirúrgico pode variar de 16\%-95\%, com uma média de 68\%.

São considerados fatores de bom prognóstico para o resultado pós-operatório: doença sem fundo compensatório, capacidade de voltar ao trabalho após a cirurgia, ausência de litigio trabalhista e uma fusão sólida do segmento operado. Por outro lado, são considerados fatores de mau prognóstico para o resultado do tratamento cirúrgico: idade avançada, doença de fundo emocional e expectativas irreais de cura. ${ }^{20,21}$

O resultado clínico do tratamento cirúrgico, no entanto, nem sempre está relacionado ao sucesso na obtenção de uma artrodese sólida, pois mesmo na presença de uma união fibrosa (pseudartrose) do segmento, os resultados podem ser tão bons quanto nos casos em que ocorre a união sólida. A união fibrosa pode permitir estabilização suficiente do segmento instrumentado e, com isso, uma melhora da dor lombar e da dor irradiada para os membros inferiores causadas pela instabilidade da coluna.

Kornblum et al. ${ }^{22}$ em um estudo comparando o resultado clínico de pacientes submetidos a descompressão e artrodese posterior com e sem consolidação da artrodese, relata $86 \%$ de resultados bons e excelentes na avaliação clínica para o grupo com consolidação e $56 \%$ de resultados bons a excelentes para o grupo sem consolidação ou com união fibrosa da artrodese posterior.

Outro estudo feito por Tsutsumimoto et al. ${ }^{23}$ comparando os resultados pós-operatórios na presença ou não de fusão sólida demonstra melhores resultados com a fusão solida do segmento. Herkowitz et al. ${ }^{17}$ também relataram bons resultados com o tratamento cirúrgico a longo prazo, mesmo nos casos com diagnóstico de pseudoartrose do segmento. Portanto, a presença ou não da consolidação da artrodese não parece ser o fator determinante para o bom resultado do tratamento cirúrgico. ${ }^{24,25}$

Diante da dificuldade na avaliação e comparação de diferentes métodos dos resultados cirúrgicos, os questionários de Oswestry e SF-36 se tornaram ferramentas que tentam quantificar, atribuindo valores numéricos, parâmetros predominantemente subjetivos. No presente estudo, os pacientes apresentam idade média de 49 anos, portanto, em plena vida produtiva. (Tabela 1) Assim, melhoraram os escores dos questionários Oswestry e componente funcional (PCS) do SF-36, apresentando evolução favorável comparando o período pré-operatório e um ano de pós-operatório. (Tabela 2)

Outro fator que tem sido apontado como possível causa de falha do tratamento cirúrgico é a sobrecarga do nível adjacente que pode ocorrer após a estabilização de um determinado segmento da coluna. A fusão solida posterior pode aumentar a chance de estresse nos segmentos adjacentes. Quando sintomática, a degeneração dos níveis adjacentes após fusão posterior pode influenciar negativamente no resultado pós-operatório e até necessitar de nova abordagem cirúrgica para correção. ${ }^{26}$

Glassman et al. ${ }^{27}$, em uma revisão dos resultados pós-operatórios de artrodese lombar, relataram uma melhora de 6,21 pontos no questionário SF-36 e melhora de 16,4\% no questionário Oswestry após dois anos de tratamento cirúrgico. Em outro estudo do mesmo autor, a avaliação de pacientes acima de sessenta e cinco anos submetidos a descompressão e artrodese posterior mostrou uma melhora de $28,5 \%$ no questionário Oswetry, indicando, assim, uma melhora da qualidade de vida dos pacientes avaliados. ${ }^{28}$

No presente estudo, encontramos resultados semelhantes ao descrito por esses trabalhos. A queda do escore no questionário Oswestry e a melhora da pontuação no componente físico (PCS) do SF-36 com significância estatística indica uma evolução favorável na qualidade de vida dos pacientes após a cirurgia de descompressão e artrodese posterolateral. (Figuras 1 e 2)

Fazendo as comparações entre os cinco momentos em que foram aplicados os questionários, observamos que o escore médio do Oswestry diminui do período pré-operatório para os demais mo- 
mentos de avaliação pós-operatório. A analise do escore médio do PCS do questionário SF-36 aumenta com significância estatística do período pré-operatório para o pós-operatório de um ano.

\section{CONCLUSÃO}

A avaliação realizada por meio dos questionários Oswestry 2.0 e do componente físico (PCS) do SF-36 nos pacientes submetidos cirurgia de descompressão e artrodese por via postero- lateral da coluna lombar para tratamento de doença degenerativa da coluna, mostrou uma evolução favorável, com melhora dos escores entre o período pré-operatório e o período de um ano de pós-operatório.

Todos os autores declaram não haver nenhum potencial conflito de interesses referente a este artigo.

\section{REFERÊNCIAS}

1. Webb R, Brammah T, Lunt M, Urwin M, AllisonT, Symmons D. Prevalence and predictors of intense, chronic, and disabling neck and back pain in the UK general population. Spine (Phila Pa 1976). 2003:28(11):1195-202

2. Andersson GB. Epidemiology of low back pain. Acta Orthop Scand Suppl. 1998;281:28-31.

3. Cassidy JD, Carroll LJ, Côté P. The Saskatchewan health and back pain survey. The prevalence of low back pain and related disability in Saskatchewan adults. Spine (Phila Pa 1976). 1998;23(17): 1860-6.

4. Kent P, Keating J. Do primary-care clinicians think that nonspecific low back pain is one condition? Spine (Phila Pa 1976). 2004:29(9):1022-31.

5. Yong-Hing K, Kirkaldy-Willis WH. The pathophysiology of degenerative disease of the lumbar spine. Orthop Clin North Am. 1983;14(3):491-504.

6. Liao JC, Chen WJ, Chen LH, Niu CC, Keorochana G. Surgical outcomes of degenerative spondylolisthesis with L5-S1 disc degeneration: comparison between lumbar floating fusion and lumbosacral fusion at a minimum 5-year follow-up. Spine (Phila Pa 1976). 2011:36(19):1600-7.

7. Suratwala SJ, Pinto MR, Gilbert TJ, Winter RB, Wroblewski JM. Functional and radiological outcomes of 360 degrees fusion of three or more motion levels in the lumbar spine for degenerative disc disease. Spine (Phila Pa 1976). 2009:34(10):E351-8.

8. Fairbank JC, Pynsent PB. The Oswestry Disability Index. Spine (Phila Pa 1976). 2000;25(22):2940-52.

9. Ware J, Snow K, Kosinski M, Gandek B. SF-36 health survey: Manual and interpretation guide. Boston, MA: The Health Institute; 1993.

10. Ware JE, Kosinski M, Keller SK. SF-36 physical and mental health summaries scales: a user's manual. Boston, MA: The Health Institute; 1994.

11. Kirkwood BR, Sterne JA. Essential medical statistics. 2nd ed. Massachusetts, USA: Blackwell Science; 2006

12. Neter J, Kutne MH, Nschtsheim CJ, Wasserman W. Applied linear statistical models. 4th ed. Chicago (IL): Richard D. Irwing; 1996

13. Bridwell KH, Sedgewick TA, O'Brien MF, Lenke LG, Baldus C. The role of fusion and instrumentation in the treatment of degenerative spondylolisthesis with spinal stenosis. J Spinal Disord. 1993:6(6):461-72.

14. Deyo RA, Nachemson A, Mirza SK. Spinal-fusion surgery - the case for restraint. N Engl J Med. 2004;350(7):722-6.

15. Maghout Juratli S, Franklin GM, Mirza SK, Wickizer TM, Fulton-Kehoe D. Lumbar fusion outcomes in Washington State workers' compensation. Spine (Phila Pa 1976). 2006;31(23):2715-23.

16. Glassman SD, Carreon L, Dimar JR. Outcome of lumbar arthrodesis in patients sixty-five years of age or older. Surgical technique. J Bone Joint Surg Am. 2010;92(Suppl 1 Pt 1):77-84.

17. Herkowitz HN, Abraham DJ. Degenerative lumbar spondylolisthesis. Semin Spine Surg. 1999;11:28-33

18. Rönnberg K, Lind B, Zoëga B, Halldin K, Gellerstedt M, Brisby H. Patients' satisfaction with provided care/information and expectations on clinical outcome after lumbar disc herniation surgery. Spine (Phila Pa 1976). 2007;32(2):256-61.

19. Turner JA, Ersek M, Herron L, Haselkorn J, Kent D, Ciol MA, et al. Patient outcomes after lumbar spinal fusions. JAMA. 1992;268(7):907-11.

20. Bernard TN Jr. Repeat lumbar spine surgery. Factors influencing outcome. Spine (Phila Pa 1976). 1993;18(15):2196-200.

21. Toyone T, TanakaT, Kato D, Kaneyama R, Otsuka M. Patients' expectations and satisfaction in lumbar spine surgery. Spine (Phila Pa 1976). 2005;30(23):2689-94.

22. Kornblum MB, Fischgrund JS, Herkowitz HN, Abraham DA, Berkower DL, Ditkoff JS. Degenerative lumbar spondylolisthesis with spinal stenosis: a prospective long-term study comparing fusion and pseudarthrosis. Spine (Phila Pa 1976). 2004;29(7):726-33

23. TsutsumimotoT, Shimogata $M$, Yoshimura $Y$ Misawa $H$. Union versus nonunion after posterolateral lumbar fusion: a comparison of long-term surgical outcomes in patients with degenerative lumbar spondylolisthesis. Eur Spine J. 2008;17(8):1107-12.

24. Mardjetko SM, Connolly PJ, Shott S. Degenerative lumbar spondylolisthesis. A meta-analysis of literature 1970-1993. Spine (Phila Pa 1976). 1994:19(Suppl 20):2256S-2265S.

25. Fischgrund JS, Mackay M, Herkowitz HN, Brower R, Montgomery DM, Kurz LT. 1997 Volvo Award winner in clinical studies. Degenerative lumbar spondylolisthesis with spinal stenosis: a prospective, randomized study comparing decompressive laminectomy and arthrodesis with and without spinal instrumentation. Spine (Phila Pa 1976). 1997;22(24):2807-12

26. Park P, Garton HJ, Gala VC, Hoff JT, McGillicuddy JE. Adjacent segment disease after lumbar or lumbosacral fusion: review of the literature. Spine (Phila Pa 1976). 2004:29(17):1938-44..

27. Glassman SD, Carreon LY, Dimar JR, Campbell MJ, Puno RM, Johnson JR. Clinical outcomes in older patients after posterolateral lumbar fusion. Spine J. 2007;7(5):547-51.

28. Glassman SD, Polly DW, Bono CM, Burkus K, Dimar JR. Outcome of lumbar arthrodesis in patients sixty-five years of age or older. J Bone Joint Surg Am. 2009;91(4):783-90 